\title{
Desequilibrios territoriales en Cartagena de Indias, análisis desde la distribución de los equipamientos ${ }^{1}$
}

Territorial imbalances in Cartagena de Indias, analysis from the distribution of equipment

\section{Resumen}

Israel Cabeza Morales ${ }^{2}$

El análisis de los equipamientos puede aportar al tratamiento de las problemáticas del desarrollo más allá de una perspectiva de sostenibilidad, por lo cual se toma el concepto de cohesión territorial como sinónimo de equilibrio, y se constituye en referente para pensar en el desarrollo de los territorios en función de sus gentes. Este tipo de ejercicios muestra, a partir de la relación entre la distribución de los equipamientos y la funcionalidad económica de algunas zonas en Cartagena de Indias, los desequilibrios territoriales que pueden ser reducidos al insertar la cohesión como herramienta para los procesos de ordenamiento territorial.

Palabras clave: cohesión social, cohesión territorial, desarrollo territorial, desequilibrio, funcionalidad urbana.

\section{Abstract}

Analysis of equipment can contribute to the treatment of development issues beyond a sustainability perspective, so it takes the concept of territorial cohesion as a synonym for balance, and is a benchmark for thinking about the development of territories depending on its people. From the relationship between the

1 Artículo derivado del proyecto de investigación "Evaluación de la cohesión territorial en Cartagena de Indias, Colombia: Un aporte desde la geografía". Grupo de investigación Vida Territorial Sostenible -VTS-.

2 Docente Ocasional, Escuela de Ciencias Sociales, Universidad Pedagógica y Tecnológica de Colombia -UPTC-. Israel. cabeza@uptc.edu.co 
distribution of equipment and economic functionality of some areas in Cartagena de Indias this type of exercise tends to show the regional imbalances that can be reduced by inserting cohesion as a tool for land use planning processes.

Keywords: Social cohesion, territorial cohesion, territorial development, Imbalance, urban functionality. 


\section{Introducción}

En el marco de la comprensión de las dinámicas territoriales, la actividad económica cumple un rol determinante para el análisis de la configuración de redes que sostienen la productividad de ciudades y regiones, lo cual generalmente está basado en una exacerbada optimización de recursos, que va desde la proximidad a cuerpos de agua a zonas de riqueza vegetal, hasta el aprovechamiento de elementos que constituyen el escenario sobre el cual se desarrollan de modo particular las relaciones entre sociedad y naturaleza. Lo anterior permite analizar los procesos y las zonas en donde se genera un desgaste progresivo, no solo desde la funcionalidad de la economía de una ciudad, sino de los desequilibrios que se dan en el interior de la misma.

La existencia en Colombia de planes y proyectos, a escala nacional, regional y local, obedece en algunos casos a temas sectoriales no articulados entre sí, y menos con su efectividad real en términos de aumento de la calidad de vida, sostenibilidad de los recursos sociales, económicos, políticos e institucionales; lo cual dificulta la inserción de los territorios en los procesos de competitividad en marcos nacionales, regionales e internacionales. Frente a este tipo de situaciones de dificultad, en cuanto a limitaciones para perspectivas de competitividad, resulta de interés el análisis de la distribución de los equipamientos de un ciudad como Cartagena de Indias, donde la tradición fuerte que se ha instaurado a través de la industria y el turismo como pilares para el desarrollo, a pesar del relativo crecimiento económico, no ha logrado generar niveles de bienestar en gran parte de sus habitantes, por cuanto:

- Los criterios de construcción del Plan de Ordenamiento Territorial (POT) han estado orientados básicamente hacia el crecimiento económico en función de la plusvalía, desconociendo los límites y posibilidades territoriales.

- La distribución espacial de los equipamientos urbanos y rurales se ha dado de manera nodal -tanto en infraestructura económica como social-, y ha restringido el acceso de la población a ciertos desarrollos o áreas específicas de interés económico, lo que ha generado la "marginalización espacial" de las poblaciones.

Por lo anterior, este ejercicio pretende llegar a la resolución del siguiente interrogante: ¿Cómo están distribuidos los equipamientos en el Distrito de Cartagena de Indias y cuál es su articulación con un desarrollo territorial cohesionado? A través de los elementos influyentes en la distribu- 
ción espacial de las principales actividades económicas en Cartagena de Indias, para lo cual fue propuesta la identificación de los aspectos determinantes de la actividad económica de la ciudad. Posteriormente se caracterizaron las relaciones entre los distintos elementos que marcan la dinámica económica de la ciudad y finalmente se determinaron problemáticas asociadas a la concentración de la actividad económica en algunos puntos de la ciudad.

El desarrollo del ejercicio se dirigió a develar la forma en que la distribución funcional de algunos equipamientos genera un ordenamiento que permite o no, la cohesión de la ciudad como sistema territorial, principalmente por la importancia que se le otorga a la especificidad socioeconómica de algunas zonas de la ciudad.

\section{Sustento teórico}

La nuevas lógicas del capital, la inserción en espacios cada vez más interconectados, el aprovechamiento del tiempo y los retos que tales circunstancias implican, hacen que el ocio, el descanso y la recreación, se reconfiguren convirtiéndose en herramientas para el aprovechamiento económico. De esta manera surge la base de la consolidación del turismo como actividad económica de gran rentabilidad (Ayuso, 2003). Este tipo de aprovechamiento generalmente desconoce la necesidad de concebir las condiciones para el desarrollo del mismo, como una relación donde debe haber cierta reciprocidad.

En términos del desarrollo -entendido este como el progreso en condiciones de bienestar-, la sostenibilidad debe responder a la comprensión de la relación directa entre sociedades y ecosistemas (Lozano, 2008); por ende debería hablarse de sociedades ecosistémicas, regidas por principios de funcionalidad que eviten en su interior cualquier actividad de tipo utilitarista, de manera que se regulen transformaciones socioespaciales que puedan tener impactos nocivos sobre un territorio.

Muchas de las transformaciones que se dan en un territorio, están asociadas a elementos como la funcionalidad y el crecimiento económico, los cuales no son necesariamente contrarios al bienestar. Sin embargo, la crítica a estos se sostiene al concebir la imposibilidad de un crecimiento económico que permita el afianzamiento de acciones de integración y subsidiariedad, que faciliten la construcción de un territorio más integrado, para lograr un buen vivir (Farah \& Vasapollo, 2011).

Hacer alusión al crecimiento económico en procesos de integración territorial, estos últimos asociados a 
vínculos generadores de equilibrio, no solo implica una inversión potencial de recursos de distinto orden, redes de conectividad, recursos naturales y humanos, que relacionan: temporalidades, transporte, distracción, sanidad, ética, materialización, servicio, funcionalidad, sostenibilidad, segregación, entre otros. Más allá de la visión economicista de este tipo de escenarios, subyace su tratamiento como un fenómeno de complejidad territorial, que contiene una amplia dimensión socioespacial, lo cual se hace particularmente interesante para el tratamiento de zonas costeras y de economías intermedias, con aspiraciones de insertarse, mediante la oferta de servicios, en el mercado mundial.

En tal sentido, el turismo, la industria o cualquier otro sector productivo, debe ser una fuente primordial para el análisis de algunos procesos regionales, particularmente en casos donde se asocia la potencialización de los mismo al aumento de la brecha socioeconómica de un territorio; es decir, cuando el avance en términos económicos evidencia una relación inversamente proporcional a las condiciones socioeconómicas de las comunidades, aumentando los desequilibrios territoriales. Por consiguiente, para el surgimiento de estrategias que posibiliten un papel articulador del desarrollo económico, con la pretensión de que la visión de desarrollo en general -más allá de lo económico-, sea pretendida en función de los territorios (Múnera, 2007).

De esta manera se articula el análisis de territorio a las nuevas dimensiones, que trascienden su formalidad como espacio geográfico y construcción cultural, y llevan a la necesidad de concebirlo como un sistema social autopoiético que implica una visión sistémica del mismo, como un elemento dinámico asociado al dialogo de la acción-comunicación con entidades fijas (Pfeilstetter, 2011). Así, se reconoce la condición reproductiva de los elementos asociados al territorio y la pertinencia de que sean abordados desde la cohesión territorial, lo que denota una relación con la sostenibilidad territorial (Gutiérrez, 2010).

\section{Cohesión territorial}

El origen del concepto de cohesión territorial se explica como una de las principales pretensiones de la Comunidad Europea, la cual emplea el término en el artículo 16 de su Tratado Constitutivo de 1957, haciendo mención a la necesidad de promover la cohesión social y territorial (Fernández et al., 2009). Posteriormente, en 1997, el artículo 7D del Tratado de Amsterdam la retoma como complemento de la cohesión económica y social, lo cual se materializaría a 
través de los Fondos Estructurales, entre ellos el Fondo de Cohesión, creado desde 1993 con el Tratado de Maastricht (Zoido et al., 2009).

La cohesión territorial se proyecta como una respuesta a la necesidad de explotar nuevas oportunidades para la afirmación de los intereses de las diversas colectividades territoriales, las cuales son entendidas desde los bloques: mercado, Estado y sociedad civil (Ferrão, 1995). Bloques que al ser integrados posibilitan configurar una nueva política regional. Cabe mencionar que la cohesión no debe ser vista únicamente como herramienta en función de la competitividad o de la integración funcional del territorio, sino como insumo para la reducción de los desequilibrios territoriales.

Algunos de los estudios más recientes respecto a la cohesión territorial, han permitido la conjugación de elementos propios de un territorio para el estudio de la cohesión en el mismo, a través de indicadores de diversa índole -en su mayoría de fuentes secundarias-, dada la complejidad que implica la interconexión e interescalaridad que requiere la cohesión territorial. Hay que reconocer trabajos como el de Fernández et $a l$. , (2009) en el que analizan la cohesión territorial asociada a un concepto de desarrollo más articulado a la funcionalidad, para la identifica- ción de desequilibrios territoriales en España. Igualmente existen ejercicios como el de Delgadillo y Cortes (2009), en los que el concepto de cohesión juega un papel implícito en el texto y su presencia en el mismo es asociada al tratamiento del desarrollo territorial, tanto desde las reflexiones teórico-conceptuales como de la propuesta de lineamientos para la puesta en marcha o elaboración de los esquemas de ordenamiento o políticas territoriales.

La integración a la que se apunta a través de la cohesión territorial, se sustenta en la articulación de la condición autopoiética del territorio y la necesidad de que en búsqueda de la conservación y funcionalidad del mismo se llegue a los conceptos de sostenibilidad territorial y particularmente al de cohesión como producto de su complejidad; es decir, del reconocimiento de diversas interrelaciones como sustento del mismo. Por lo cual se hace necesario propender al establecimiento y conservación de vínculos que posibiliten la actuación sobre los territorios de manera conjunta, para lograr así la construcción de nuevas realidades territoriales. De allí que se asocie la cohesión con elementos como el desarrollo equilibrado, el acceso a equipamientos, servicios y vivienda, y la articulación tanto interna como externa en un territorio (Hildenbrand, 2009). 
En Colombia, el desarrollo territorial sostenible es el concepto que se ha usado para apoyar la capacidad administrativa frente a la gestión y manejo de los recursos territoriales, cuya referencia -para el caso de Cartagena de Indias, en el Plan de Ordenamiento Territorial (POT)- es mínima, en cuanto a conceptos como sostenibilidad y desarrollo territorial, sin tenerse claridad de las pretensiones subyacentes a dichos conceptos. Este documento es producto de un ejercicio en el marco de una investigación en la que, a diferencia de las antes mencionadas, se pretende establecer la necesidad de concebir la cohesión territorial como un deber ser del ordenamiento.

Se sostiene la pertinencia de la conexión entre los bloques, que serían las unidades de análisis relevantes para la cohesión territorial: lo económico y lo social, que desde una perspectiva de sostenibilidad territorial reconoce que: "The principles of sustainable development call for an integration of information about economic environmental, and social factors in decision making"3 (Kelly, 1998, p. 453). La articulación entre los bloques ya mencionados es dependiente del componente material que sirve de insumo para la consolidación de un tipo de relación entre sociedad-naturaleza,

3 Los principios del desarrollo sostenible llaman a la integración de información sobre los factores económicos, medioambientales y sociales para la toma de decisiones (Kelly, 1998, p. 453). de allí la importancia de los equipamientos.

Para el tratamiento de la distribución espacial de los equipamientos, se parte de que el desarrollo económico se ve favorecido por la especialización territorial, la cual, según Ferrão (1998), obedece a la compresión espacio-temporal de los lugares estratégicos, genera eficacia económica y desencadena simultáneamente inequidad territorial -traducida en problemas de orden ambiental tanto a nivel urbano como rural-. Estas problemáticas van más allá de lo superficial, cuya causalidad es explicada por Foladori (2001) como producto de la continuidad del proceso de industrialización, o según Harvey (2004), como una acumulación por desposesión, bastante avanzada en el caso de la industria y similarmente contundente con las recién consolidadas iniciativas de la generación de capital, como el turismo.

Sin duda alguna, el gran descubrimiento alrededor de los estudios económicos y académicos, ha sido la comprensión de que el sistema económico es el responsable de consolidar las ciudades como centros productivos desde su interior (Foladori, 2001). Esto, sumado a la preocupación por el deterioro de las condiciones del ambiente, pero especialmente por el desarrollo en función de los territorios, se constituye en el principal reto de las sociedades actuales. 
Ante tal situación, elordenamientoterritorial no debe combatir la polarización generada por la eficiencia económica, sino tender a un equilibrio razonable entre equidad, distribución espacial y costo, en términos de crecimiento global. Lo cual, en otras palabras, sería apuntar a la funcionalidad sistémica de los territorios, llevando implícito el principio de sostenibilidad. Este último no dista de la cohesión territorial, que se constituye en complemento para la reorientación de ordenamiento territorial, como hasta ahora ha sido concebido en Colombia. De esta manera, y sumada la distribución, los elementos que hacen parte de los distintos sectores económicos de un territorio, el uso del suelo y la lógica sobre la cual se proyecta su estructura vial, es posible llegar a una visión más completa del nivel de articulación dentro de estos elementos tan determinantes para la dinámica económica, así como para la gobernanza y, por ende, para la cohesión.

\section{Metodología}

Para analizar los indicadores de equipamientos tomados de la Secretaría de Planeación Distrital (2007), fue necesario agruparlos como aparecen en la Tabla 1.

Tabla 1. Agrupación de equipamientos.

\begin{tabular}{|c|c|}
\hline \multicolumn{2}{|c|}{ Grupos de indicadores } \\
\hline Equipamiento básico \\
- & Hospitales \\
- & Estegios \\
\hline Equipamiento de desarrollo \\
- & Universidades \\
- & Estación de Servicio \\
- & Centros Comerciales \\
- & Antenas \\
\hline Equipamiento para el esparcimiento \\
- $\quad$ Canchas \\
- Iglesias \\
\hline Equipamiento de seguridad \\
- $\quad$ Estaciones de Policía \\
- $\quad$ Cámaras de seguridad
\end{tabular}

Fuente: Secretaria de Planeación Distrital (2007) 
Con la agrupación de acuerdo con la tipología de los indicadores, se logró establecer un conjunto de equipamiento según sus características, lo cual enriqueció el análisis por localidad. Esto fue apoyado a partir de la explicación de problemas que tienen injerencia en la realidad ambiental de la ciudad -contrastados en campo-, para facilitar la asociación de algunas características por localidad. Así se llegó a una reflexión alrededor de los desequilibrios que sobre algunas de las localidades se ejercen a través de los equipamientos y su trascendencia en la perspectiva de sostenibilidad para el Distrito.

Cabe resaltar que la agrupación se estableció a partir de la información obtenida por la Secretaría de Planeación Distrital y que el componente de vías no se incluyó por las limitaciones del ejercicio y consolidación de la información a la fecha de realización del mismo.

\section{Caracterización del equipamiento en Cartagena de indias}

\subsection{La ciudad distrito}

Cartagena de indias es una ciudad que se ha posicionado como turística, de gran reconocimiento en Colombia y el resto del mundo; una ciudad que la UNESCO ha declarado desde 1985 patrimonio cultural de la humanidad y a la cual se le ha otorgado el título de Distrito Turístico y Cultural desde 1987, reafirmado por la Constitución Política de 1991. El Distrito tiene una extensión de 709,12 km² y una amplia diversidad social entre sus 944.250 habitantes, según proyección para el año 2010 , de los cuales 45.050 se encuentran por fuera de la cabecera municipal, sin mencionar el $36 \%$ del total de la población, que corresponde a afrodescendientes (DANE, 2005).

En la ciudad se han generado iniciativas económicas de gran significación para la actividad turística e industrial, con grandes desequilibrios sociales manifestados en toda su extensión. Por esta razón se pretende generar información desde las tres localidades (Histórica y del Caribe Norte LHCN; de la Virgen y Turística LVT; e Industrial de la Bahía LIB) que conforman la ciudad, así como las unidades comuneras rurales y urbanas de gobierno, contenidas dentro de cada localidad, incluyendo los quince corregimientos, once caseríos y un centro poblado que, junto con la cabecera municipal, conforman el Distrito como unidad territorial, en concordancia con el Decreto No. 0977 del 2001, por el cual se establece el POT del Distrito (Alcaldía mayor, 2001).

La ciudad puede considerarse como un conjunto de minirregiones, dada la fragmentación generada por la especialización económica de algunas 


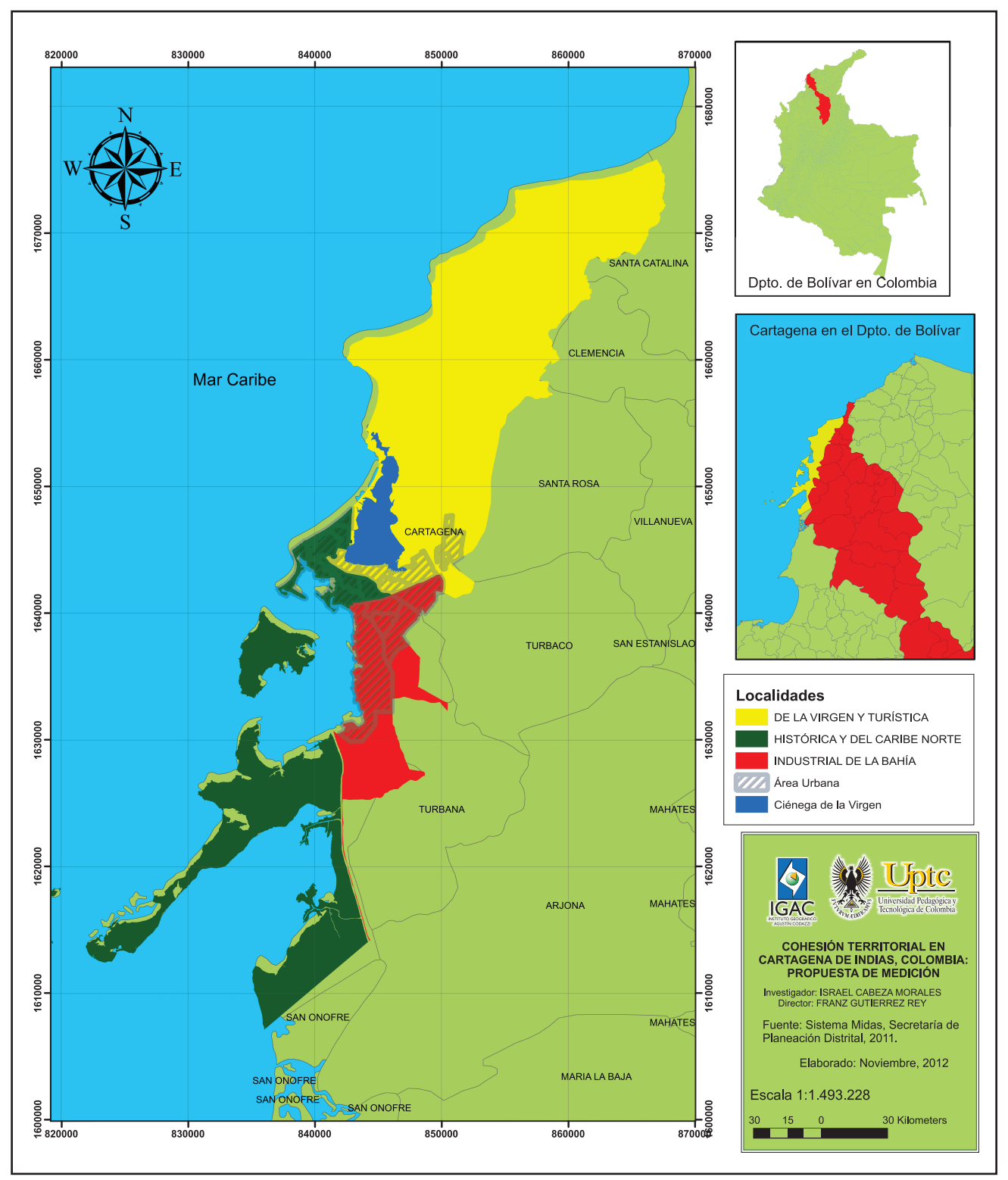

Figura 1. Localidades de Cartagena de Indias.

Fuente: Secretaría de Planeación Distrital (2007).

zonas; en su interior pueden defi- con una extensión de $100 \mathrm{~km}^{2}$, en nirse como sectores: por un lado el torno al cual se encuentran zonas centro histórico, que sobresale por como los barrios Bocagrande, el Lasu carácter turístico y comercial, guito y Castillo grande; que además 
de consolidar una de las zonas residenciales de mayor valor económico en la ciudad, también concentra gran parte de la actividad hotelera y turística por su proximidad al mar y por las alternativas comerciales que ofrece. Por otro lado, el sector industrial, asentado principalmente en la zona de Mamonal, se comunica con el corredor industrial del Bosque, el cual conecta la ciudad con la sociedad portuaria establecida en la isla de Manga, un barrio que recoge un poco de la ciudad (mezclando lo residencial de estrato alto con medio y un bajo casi inexistentes, a punto de ser exterminados por proyectos urbanísticos de gran nivel).

Igualmente existen otros sectores como: Barú y la Boquilla, dos de los quince corregimientos que según POT hacen parte del sector rural del distrito; alrededor de Cartagena existen espacios transicionales en los que no hay más que distancias espaciales y económicas, que denotan pobreza $\mathrm{y}$ funcionalidad social operativa, que no permiten la construcción de tejido social unificado en la ciudad. No hay una construcción territorial aglutinante de la población, pues se está apuntando a la fragmentación total de la ciudad, ya que mientras esta continúa creciendo, la gente se ve aislada de algunos espacios. Se está tratando de idear una funcionalidad más productiva en términos del sistema económico imperante; lo úni- co que se legitima es que la ciudad necesita venderse a como dé lugar, empleando la inversión extranjera como herramienta para superar las desigualdades sociales.

Así, el espacio de la ciudad ha sido visionado por la clase dirigente nacional y local, a lo largo del siglo $\mathrm{XX}$, como un escenario potencial para el desarrollo económico y social, a través de la legitimación en el subconsciente de la población cartagenera, que la ciudad es más que murallas, y trascendiendo esta idea, pasa a ser hoy ya centro histórico para los cartageneros, y el corralito, como lo denominan cientos de turistas; lo cual marca un paso para la instauración de núcleos de diferentes actividades económicas.

Prueba de ello es que desde finales del siglo XIX -a causa de una crisis de exportaciones, durante el Gobierno del cartagenero Rafael Núñez como presidente del país-, aparecen las primeras fábricas en Cartagena y en el estado soberano de Bolívar (actual departamento del cual Cartagena es capital), fábricas de driles, hilados, laboratorios químicos, y posteriormente la construcción del ferrocarril, el mercado y el matadero público.

Con la crisis de los primeros años del siglo XX, la industria naciente se esfuerza por sostenerse y dar frutos, 
pero es hacia 1957 cuando se consolida Mamonal y la zona industrial del Bosque, y desde los años sesenta, el patrimonio natural y cultural se utilizó como base para desarrollar el turismo y convertir el Distrito como el centro nacional e internacional de turismo más destacado en Colombia a finales del siglo XX (Báez \& Calvo, 1999).

Este y otros aspectos -especialmente la autonomía como Distrito, gracias a la Constitución de 1991-, le han permitido impulsar proyectos de crecimiento económico que han reconfigurado a Cartagena como ciudad, haciéndola un sitio atractivo para turistas, inversionistas, desplazados y familias en búsqueda de un mejor futuro; lo que sustenta sus retos en materia de cohesión territorial, debido a los problemas sociales que enfrenta.

\subsection{Agrupación de los equipamientos}

La síntesis de la información agrupada es mostrada en la Tabla 2 y la Figura 2; las cuales son la recopilación de este ejercicio de aproximación al nivel de integración o bienestar que pueden generar algunos equipamientos.

Tabla 2. Equipamiento Distrital

\begin{tabular}{|c|c|c|c|c|}
\hline $\begin{array}{c}\text { Tipo de } \\
\text { equipamiento/Localidad }\end{array}$ & LHCN & LVT & LIB & $\begin{array}{l}\text { Distrito de } \\
\text { Cartagena de } \\
\text { Indias }\end{array}$ \\
\hline \multirow{2}{*}{ Seguridad } & 72 & 19 & 15 & 106 \\
\hline & 67,92 & 17,92 & 14,15 & 100 \\
\hline \multirow{2}{*}{ Esparcimiento } & 125 & 68 & 82 & 275 \\
\hline & 45,45 & 24,73 & 29,82 & 100 \\
\hline \multirow{2}{*}{ Desarrollo } & 90 & 12 & 33 & 135 \\
\hline & 66,67 & 8,89 & 24,44 & 100 \\
\hline \multirow{2}{*}{ Básicos } & 254 & 145 & 156 & 555 \\
\hline & 45,77 & 26,13 & 28,11 & 100 \\
\hline \multirow{2}{*}{ Total } & 541 & 244 & 286 & 1071 \\
\hline & 50,51 & 22,78 & 26,70 & 100 \\
\hline
\end{tabular}

Fuente: Secretaría de Planeación Distrital (2007).

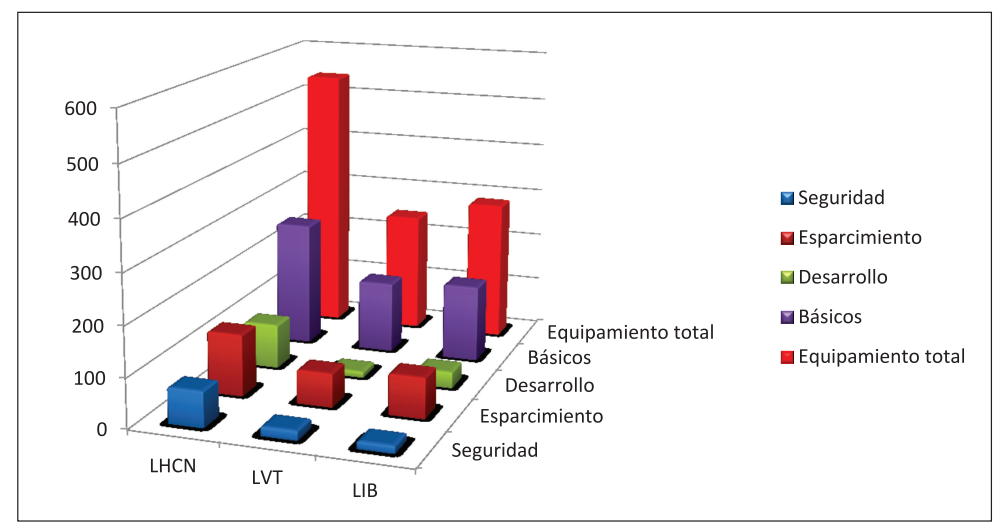

Figura 2. Equipamiento Distrital 
Desde la revisión del equipamiento total, sobresale la diferencia entre la LHCN, superando en gran proporción a la LIB y la LVT, respectivamente, mientras entre estas últimas la diferencia es mínima (ver Tabla 2). Esto se explica porque la LHCN, a pesar de ser la segunda localidad en área, se constituye en la de mayor población y diversidad de condiciones sociales, debido a que en su interior se alberga el casco histórico de la ciudad y todo el complejo comercial (donde se reúnen el sector político institucional, el turístico, entre otros). Para explicar la particular dinámica de esta localidad, hay que resaltar que en ella se concentra la principal zona de playa, el puerto aéreo, la bahía y la ciénaga de la Virgen -principal cuerpo de agua dulce- que separa al casco histórico y zonas ampliamente turísticas -como Bocagrande y el Laguito- de zonas de aguda situación social -como los barrios: Daniel Lemaitre, Torices, y San Francisco, entre otros- (ver Tabla 3).

Tabla 3. Población y viviendas por localidades

\begin{tabular}{|c|c|c|c|c|c|c|c|c|}
\hline & & & \multicolumn{6}{|c|}{ Número de viviendas por estrato } \\
\hline & Población & $\begin{array}{c}\text { Número de } \\
\text { viviendas }\end{array}$ & $\begin{array}{c}\text { Estrato } \\
1\end{array}$ & $\begin{array}{c}\text { Estrato } \\
2\end{array}$ & $\begin{array}{c}\text { Estrato } \\
3\end{array}$ & $\begin{array}{c}\text { Estrato } \\
4\end{array}$ & $\begin{array}{c}\text { Estrato } \\
5\end{array}$ & $\begin{array}{c}\text { Estrato } \\
6\end{array}$ \\
\hline LHCN & 388,489 & 63,694 & 10,680 & 17,590 & 13,895 & 5,686 & 6,154 & 5,842 \\
\hline LVT & 334,383 & 49407 & 25,873 & 13,561 & 3,576 & 1,225 & 0 & 0 \\
\hline LIB & 346,883 & 214,069 & 131,307 & 73,029 & 9,697 & 15 & 21 & \\
\hline
\end{tabular}

Fuente: Secretaría de Planeación Distrital (2007).

En la anterior tabla se evidencia el gran desequilibrio que hay en la distribución de la población, según condiciones sociales en la LHCN. Como lo refleja la Tabla 2, existe un factor determinante que marca la dinámica de esta localidad de gran importancia para la ciudad, pues en ella se encuentra el $50 \%$ del total de los equipamientos de esta, y cuya distribución en el interior de la localidad no alcanza a ser proporcional con la dimensión de la misma.
Lo anterior se traduce en una presión exacerbada sobre la LHCN, que además de contener el casco histórico de la ciudad, aún funciona como principal centro de actividades socioculturales, económicas y políticas. En el interior de la LHCN se encuentra la zona del barrio el Bosque "corredor de carga" (eje vial que conecta la zona industrial de Mamonal con la Sociedad Portuaria), por donde transita más del $85 \%$ de los containers que salen o llegan a la ciudad a tra- 
vés del puerto, ubicado en la isla de Manga, y donde hay un peaje vial con el fin de mantener el corredor, lo cual transforma las condiciones de movilidad en el interior de la ciudad para propios y visitantes; además del riesgo que implica la presencia del corredor de carga dentro de una zona que desde sus inicios ha sido eminentemente urbana y que a la fecha, a pesar de rodearse de algunas bodegas y empresas de diversa naturaleza, sigue siendo un sector donde prima la propiedad residencial.

La presión ejercida afecta el principal cuerpo de agua en el interior de la ciudad, la ciénaga de la Virgen, que por estar cerca a la Sociedad Portuaria y tener un límite directo con la principal plaza de mercado de la ciudad, genera problemas de contaminación asociados al manejo de residuos sólidos, entre otros. Ello puede sobredimensionarse aclarando la magnitud poblacional de los principales barrios marginales por los que circunda el conjunto de caños afluentes de la misma; así como las amplias zonas de contacto directo con el perímetro de la ciénaga, las cuales forman parte de la LVT. Allí, debido al desplazamiento y los desequilibrios propios de la dinámica económica y social de la ciudad, crece la vivienda informal de tipo palafítica sobre la ciénaga, la que desencadena problemas asociados a la miseria, entre otros.

Los datos de la Tabla 4 permiten una aproximación a la presión ejercida sobre la ciénaga, datos poblacionales de algunos de los barrios marginales, particularmente aquellos que tienen mayor contacto con este cuerpo de agua.

Tabla 4. Barrios con mayor población LVT y LHCN, colindantes con la ciénaga de la Virgen.

\begin{tabular}{|l|l|l|l|}
\hline \multicolumn{2}{|c|}{ LHCN } & \multicolumn{2}{c|}{ LVT } \\
\hline San Francisco & 23,415 & Olaya Herrera (Com 5) & 45,924 \\
\hline Torices & 19,199 & El Pozón & 47,644 \\
\hline Daniel Lemaitre & 10,254 & La María & 18,323 \\
\hline Bosque & 19,046 & & \\
\hline
\end{tabular}

Fuente: Secretaría de Planeación Distrital (2007).

La localidad de la Virgen y Turística se constituye en un referente de gran importancia para explicar el comportamiento de la relaciones sociedad- naturaleza, o el requerimiento de los recursos naturales que hacen sus habitantes. Hay que partir del hecho que los equipamientos de desarrollo 
y los básicos son mínimos para el caso de esta localidad, a pesar de que su diferencia en tamaño poblacional con la LHCN no es relevante, como sí lo es su composición por estratos donde se logra constatar que, en caso de hablar de una localidad de obreros, sin duda alguna, es esta la que se destaca (Tabla 3).

Esta localidad, además de ser la de mayor límite o contacto directo con la ciénaga de la Virgen, es predominantemente residencial, pero no cuenta con insumos suficientes para jalonar el avance de las comunidades. En su interior hay barrios como Boston, Olaya Herrera y el Pozón, entre otros, cuya población es bastante numerosa (Tabla 4), lo que dificulta una relación armónica con el medio. Un ejemplo puntual consiste en la relativa apropiación de la ciénaga que se da en zonas colindantes con barrios marginales -como los anteriormente mencionados- donde debido a sus facilidades de acceso, llega mucha población indigente o desplazada que ocupa palafitos, lo cual altera cada vez el comportamiento de la ciénaga, desacelerando la extensión del mangle y aumentando los procesos de contaminación (dadas las condiciones de insalubridad en las que deben vivir).

Igualmente, la LVT cuenta con la mayor parte de las zonas rurales de la ciudad, donde no se han logrado establecer iniciativas solidas económico-productivas que posibiliten transformar las condiciones de vida de la población; con excepción del corregimiento de la Boquilla, que se ha consolidado como un sector de expansión para el turismo. Ello ha desatado cambios drásticos en los precios de suelo, y conflictos por la tenencia de la tierra, a los cuales se asocian transformaciones en la vegetación de la zona -donde predominaba el mangle-, y desplazamiento de población. Esta situación se ha visto agudizada debido a algunos proyectos viales, que están próximos a la zona de playa.

La LIB, a pesar de ser la localidad de menor tamaño, cuenta con una población mayor a la de la LVT, con un mayor número de viviendas y un menor número de población incluida en el SISBEN ${ }^{4}$. Esto se explica directamente por su nombre: Localidad Industrial y de la Bahía, que sirve tanto de canal o puente entre la LHCN y la LVT, como también se encarga de mantener un sector significativo de la economía local (industria), de ahí que también sea esta la localidad de menor población rural (Figura 3). Debido a la consolidación de la zona como industrial, en esta localidad se han gestado iniciativas barriales a modo de invasión,

4 En Colombia, SISBEN es el sistema de identificación y clasificación de potenciales beneficiarios para programas sociales. 
generándose asentamientos cuya subsistencia, a grandes rasgos, se deriva de la tranquilidad de un obrero por residir cerca de su lugar de trabajo, como sucede en el sector de
Membrillal en el corregimiento de Pasacaballos y la zona de Henequén, donde hasta hace algunos años funcionaba un botadero de basura, que hizo las veces de relleno sanitario.

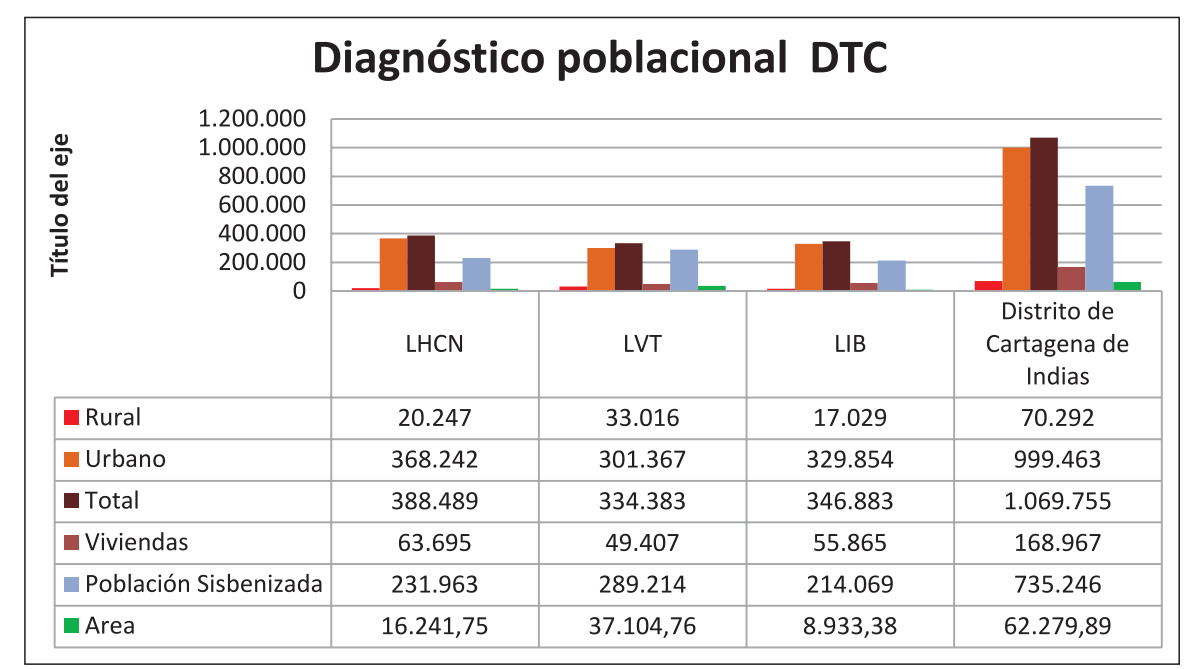

Figura 3. Diagnóstico poblacional del Distrito.

Fuente: Secretaría de Planeación Distrital (2007).

Igualmente, la propagación de los barrios marginales mediante invasión en las zonas aisladas de esta localidad, ha permitido la legitimación de esta en barrios como Arroz Barato y Puerta de Hierro. Esto explica el hecho de que la LIB tenga mayor número de equipamientos básicos que la LVT, así como en datos de equipamiento para el desarrollo, se dupliquen sus posibilidades con respecto a la LVT.

El bajo nivel de equipamiento de seguridad en la LIB, está sustentado en la poca indigencia de esta zona que está aislada del centro de la ciudad; tal situación es generada a partir de la conservación del carácter industrial de la misma y el rol que este sector económico ha propiciado, con la consolidación de zonas cercanas como espacios de residencia para población de los estratos 1 y 2 (Tabla 3 ).

De la tabla puede deducirse la importancia de un área organizada en pro de la administración pública, pues dada la clara estratificación de esta localidad, se marca un orden particular, donde las demandas de 
manejo ambiental de las comunidades desencadenan una crisis en cuanto al uso de los recursos naturales. Esta zona (al igual que las otras dos) tiene un uso diferente de las aguas que circundan su perímetro, que para el caso de la ciénaga de la Virgen, donde gran parte de este colinda con el cuerpo de agua, se ha empleado como botadero de basuras, entre otros.

\section{Resultados}

Algunos de los elementos a los que nos permite llegar el análisis de los equipamientos en el distrito de Cartagena, son los siguientes:

El hecho de que una de las tres localidades de la ciudad concentre el 50 $\%$ de los equipamientos, es la principal prueba del grado de desequilibrio de la ciudad y por ende, de las dificultades para hablar de cohesión en ella.

La presión ejercida sobre el ambiente es mayor en las localidades de gran densificación y con contraste social; las cuales, a pesar de su complejidad, no logran insertar en sus esquemas de funcionalidad, población de condiciones adversas.

Los cuerpos de agua del distrito son elementos determinantes para la dinámica urbana, y de la regulación de su perímetro depende el equilibrio de la ciudad, no solo en términos ambientales sino en principios de bienestar social.

No se ha regulado la consolidación del sector industrial, por lo cual este ha actuado de manera significativa en la redefinición urbana en el distrito, generando grandes desigualdades $\mathrm{y}$ focos de propagación de pobreza.

La especificidad económica de las localidades ha marcado una estructura social diferente dentro de cada una de ellas, y potenciado problemas derivados de la concentración desigual de dinámicas de diverso orden dentro de cada localidad.

Para consolidar un análisis más completo sobre la cohesión territorial, es conveniente incluir el equipamiento vial de la ciudad.

En el caso de una ciudad como Cartagena, donde los procesos de fragmentación urbana son evidentes, es necesario reorientar aspectos como la distribución de los equipamientos hacia un desarrollo territorial cohesionado, por lo cual urge ahondar en cuestionamientos sobre la pertinencia de que las localidades conserven una funcionalidad particular para la actividad económica, así como en posibles lineamientos para que desde los procesos de gobernanza se puedan garantizar mecanismos y es- 
cenarios de integración que permitan una construcción territorial conjunta. Igualmente es conveniente hacer estudios de segregación en el interior de una ciudad como Cartagena para descifrar los procesos que dificultan la cohesión social dentro de ella.

\section{Conclusiones}

La evaluación del grado de funcionalidad de nuestros territorios debe adoptarse como una tarea continua, cuyas herramientas deben fluctuar ajustándose a las necesidades de las sociedades. Esta debe ser una preocupación para los geógrafos y demás interesados en los fenómenos socioespaciales. Si en un momento primó el interés por el posicionamiento en el marco global, hoy la principal preocupación debe ser la búsqueda de estrategias conducentes a sociedades más equilibradas y sostenibles, donde sea posible el bienestar.

Por lo anterior, para el alcance de niveles de cohesión cada vez más armónicos dentro de la ciudad, deben impulsarse estrategias que propendan a equilibrar las condiciones sociales y económicas sobre cada localidad. Igualmente, este ejercicio sobre equipamientos permite validarse como una necesidad para cualquier pretensión de medición de cualquier elemento o indicador de gran envergadura asociado al desa- rrollo territorial, en el cual no basta trabajar una escala municipal, sino una local, que demuestre la relación de algunos elementos que definen microdinámicas territoriales, por lo cual la localidad es un buen referente para un análisis de cohesión territorial, siempre y cuando no se deje de lado la dimensión regional, que puede otorgar a los límites externos de las localidades perspectivas de interés para el estudio a escala municipal o distrital, a lo cual la infraestructura vial debe vincularse como insumo de vital importancia.

Las configuraciones generadas a partir de elementos sociales y económicos, permiten la posibilidad del análisis particular de cada uno de estos ámbitos como generadores de escenarios o ambientes de integración, por lo cual es válido hacer un análisis detallado en los niveles de cohesión que se gestan en el interior de cada uno ellos, llegando así a la pertinencia de la cohesión económica y social para la comprensión de las dinámicas territoriales y poder dirigir de mejor manera los procesos de ordenamiento territorial, hacia la generación de bienestar.

A pesar de lo significativo del análisis de los equipamientos en Cartagena de Indias, para circunscribir tal comprensión en los marcos de la cohesión territorial, es necesaria la inclusión de aspectos como la gober- 
nanza o la aprehensión, que actúan como aspectos determinantes en lo social y en lo económico, como hilos conductores de cualquier acción territorial. Con base en lo anterior se recomienda la inclusión de otros ele- mentos como la infraestructura vial, los patrones socioculturales, e incluso condiciones biofísicas, las cuales develan la endogeneidad de un territorio, más allá de los asociados a la dinámica económica.

\section{Referencias}

Alcaldía Mayor de Cartagena de Indias. (2001). Decreto No. 0977 de 2001. Recuperado de http://www.epacartagena.gov.co/doc/pot.pdf

Ayuso, S. (2003). Gestión sostenible de la industria turística: retórica y práctica en el sector hotelero español. Tesis de doctorado en Geografía, Departamento de Geografía, Universidad Autónoma de Barcelona, España. http://www.tesisenred.net/bitstream/ handle/10803/4954/sas1de4.pdf? sequence $=1$

Báez, J. \& Calvo, H. (1999). La economía de Cartagena en la segunda mitad del siglo XX: diversificación y rezago. Recuperado de http://caribe.utadeo.edu.co/dependencias/ publicaciones/pdf/economiactgena.pdf

Delgadillo, J. \& Cortés, H. (2009). Desarrollo sostenible y cohesión territorial. Recuperado de http://www.urb-al3.eu/uploads/documentos/Desarrollo_sostenible_y_cohesion_ territorial_DELGADILLO_1.pdf

Departamento Administrativo Nacional de Estadística -DANE- (2005). Perfil de Cartagena de Indias. Recuperado de http://www.dane.gov.co/files/censo2005/PERFIL_PDF_ CG2005/13001T7T000.PDF

Farah, I. \& Vasapollo, L. (Coord.) (2011). Vivir bien: ¿Paradigma no capitalista? La Paz: Universidad Mayor de San Andrés UMSA.

Fernández, A., Pedregal, B., Rodríguez, J. C., Pita, M. F. \& Zoido, F. (2009). El concepto de cohesión territorial. Escalas de aplicación, sistemas de medición y políticas derivadas. Boletín de la A.G.E., (50), 157-172.

Ferrão, J. (1995). Colectividades territoriales y globalización: contribuciones para una nueva acción estratégica de emancipación. Estudios Regionales, (43), 101-116.

Ferrão, J. (1998). Red urbana, instrumento de equidad, cohesión y desarrollo. Eure, 24(71), 0-0. Recuperado de http://www.redalyc.org/articulo.oa?id=19607105

Foladori, G. (2001). Los problemas ambientales urbanos y sus causas. Revista Paranaense de Desenvolvimento, (100), 71-80. Recuperado de http://www.ipardes.gov.br/pdf/ revista_PR/100/foladori_01.pdf

Gutiérrez, F. (2010). Organización territorial, desarrollo sostenible y nuevas visiones sobre el territorio en Colombia (1991-2010). Perspectiva Geográfica, (15), 239-260. 
Harvey, D. (2004). El nuevo imperialismo. Madrid: Akal.

Hildenbrand, A. (2009). El modelo policéntrico y compacto- la apuesta de la planificación territorial por un desarrollo urbano sostenible (ppt). En Curso Modelo de ciudad desde la perspectiva de la planificación territorial y urbanística. Universidad de Almería. Recuperado de www.ub.edu/.../Conferencia\%20Andreas\%20Hildenbrand.pdf

Kelly, K. (1998). A systems approach to identifying decisive information for sustainable development. European Journal of Operational Research, (109), 452-464. Recuperado de http://www.china-sds.org/kcxfzbg/addinfomanage/lwwk/data/A \% 20 systems \%20approach \%20to \% 20identifying \%20decisive \%20information \%20for \% 20 sustainable $\% 20$ development.pdf

Lozano, S. (2008). Procesos sociales y desarrollo sostenible: un ámbito de aplicación. Recuperado de http://upcommons.upc.edu/revistes/bitstream/2099/7077/1/lozano.pdf

Múnera, M. C. (2007). Resignificar el desarrollo. Medellín: Universidad Nacional de Colombia.

Pfeilstetter, R. (2011). El territorio como sistema social autopoiético. Pensando en alternativas teóricas al "espacio administrativo" y a la "comunidad local". Perifèria, (14), 1-17. Recuperado de http://ddd.uab.cat/pub/periferia/18858996v14a5.pdf

Secretaría de Planeación Distrital de Cartagena. (2007). Información de soporte del sistema MIDAS. Sisben, población y equipamientos por localidades. Cartagena: División de Sistemas de Información Geográfica.

Zoido, F., Pedregal, B., Pita, M. F., Torres, F. J. \& Fernández-Tabales, A. (2009). Medición del desarrollo territorial en las escalas de detalle. Aplicación a Andalucía. En J. Farinós, J. Romero \& J. Salom (Eds.) Cohesión e inteligencia territorial (pp. 245-264). Valencia, España: Universitat de Valencia.

Recepción: 11 de febrero de 2013

Evaluación: 07 de marzo de 2013

Aprobación: 08 de abril de 2013 